\title{
ON THE DISCREPANCY OF RANDOM WALKS ON THE CIRCLE
}

\author{
Alina Bazarova $^{1}$ — István Berkes ${ }^{2}$ - MARKo RASETA $^{3}$ \\ ${ }^{1}$ Institute for Biological Physics, University of Cologne, Köln, GERMANY \\ ${ }^{2}$ Rényi Institute of Mathematics, Budapest, HUNGARY \\ ${ }^{3}$ Research Institute for Primary Care and Health Sciences and Research Institute, \\ University of Keele, Staffordshire, UNITED KINGDOM
}

\begin{abstract}
Let $X_{1}, X_{2}, \ldots$ be i.i.d. absolutely continuous random variables, let $S_{k}=\sum_{j=1}^{k} X_{j}(\bmod 1)$ and let $D_{N}^{*}$ denote the star discrepancy of the sequence $\left(S_{k}\right)_{1 \leq k \leq N}$. We determine the limit distribution of $\sqrt{N} D_{N}^{*}$ and the weak limit of the sequence $\sqrt{N}\left(F_{N}(t)-t\right)$ in the Skorohod space $D[0,1]$, where $F_{N}(t)$ denotes the empirical distribution function of the sequence $\left(S_{k}\right)_{1 \leq k \leq N}$.
\end{abstract}

Communicated by Friedrich Pillichshammer

Dedicated to Professor Radhakrishnan Nair on the occasion of his 60th birthday

\section{Introduction}

Let $X_{1}, X_{2}, \ldots$ be i.i.d. absolutely continuous random variables and let $S_{k}=$ $\sum_{j=1}^{k} X_{j}(\bmod 1)$. Then $S_{k}, k=0,1, \ldots$ is a random walk on the circle and by a classical result of Lévy [6], the distribution of $S_{k}$ converges weakly to the uniform distribution on $(0,1)$. Schatte [8] proved that the speed of convergence

(c) 2019 BOKU-University of Natural Resources and Life Sciences and Mathematical Institute, Slovak Academy of Sciences.

2010 Mathematics Subject Classification: 11K38, 60G50, 60F17.

Keywords: i.i.d. sums mod 1; empirical distribution; discrepancy; weak convergence.

Research supported by NKFIH Grant K 125569.

Licensed under the Creative Commons Attribution-NC-ND 4.0 International Public License. 


\section{ALINA BAZAROVA — ISTVÁN BERKES - MARKO RASETA}

is exponential, and letting

$$
\left.D_{N}^{*}:=\sup _{0 \leq a<1} \mid \frac{1}{N} \sum_{k=1}^{N} I_{(0, a)}\left(S_{k}\right)-a\right) \mid \quad(N=1,2, \ldots)
$$

denote the star discrepancy of the sequence $\left(S_{k}\right)_{1 \leq k \leq N}$, he also proved in [9] the law of the iterated logarithm

$$
\limsup _{N \rightarrow \infty} \sqrt{\frac{N}{\log \log N}} D_{N}^{*}=\gamma \quad \text { a.s. }
$$

where

$$
\gamma=\sup _{x \in[0,1)} \sigma^{2}(x)
$$

with

$$
\sigma^{2}(x)=x-x^{2}+2 \sum_{j=1}^{\infty}\left(\mathbb{E} I_{(0, x)}(U) I_{(0, x)}\left(U+X_{j}\right)-x^{2}\right) .
$$

Here $U$ is a uniform $(0,1)$ random variable independent of the sequence $\left(X_{n}\right)_{n \geq 1}$ and for $0 \leq a<b \leq 1, I_{(a, b)}$ denotes the indicator function of $(a, b)$, extended with period 1 . Letting

$$
F_{N}(t)=F_{N}(t, \omega)=\frac{1}{N} \sum_{k=1}^{N} I_{(0, t)}\left(S_{k}\right) \quad(0 \leq t \leq 1)
$$

denote the empirical distribution function of the first $N$ terms of the sequence $\left(S_{n}\right)_{n \geq 1}$, Berkes and Raseta [2] proved a Strassen type functional LIL for $F_{N}(t)$, yielding precise asymptotics for several functionals of the empirical process. The purpose of the present paper is to prove the following result, determining the limit distribution of $\sqrt{N} D_{N}^{*}$.

TheOREM 1. Let $X_{1}, X_{2}, \ldots$ be i.i.d. random variables and assume $X_{1}$ is bounded with bounded density. Let

$$
\Gamma(s, t)=s(1-t)+\sum_{k=1}^{\infty} \mathbb{E} f_{s}(U) f_{t}\left(U+S_{k}\right)+\sum_{k=1}^{\infty} \mathbb{E} f_{t}(U) f_{s}\left(U+S_{k}\right),
$$

where $U$ is a $U(0,1)$ variable independent of $\left(X_{n}\right)_{n \in \mathbb{N}}$ and $f_{s}=I_{(0, s)}-s$. Then the series in (41) are absolutely convergent and

$$
\sqrt{N} D_{N}^{*} \stackrel{d}{\longrightarrow} \sup _{0 \leq t \leq 1}|K(t)|
$$

where $K(s)$ a mean zero Gaussian process with covariance function $\Gamma(s, t)$.

Actually, Theorem 1 will be deduced from a more general functional result describing the weak limit behaviour of the empirical distribution function $F_{N}(t)$. 
TheOREM 2. Under the conditions of Theorem 1 we have

$$
\sqrt{N}\left(F_{N}(t)-t\right) \stackrel{D[0,1]}{\longrightarrow} K(t) \quad \text { as } \quad N \rightarrow \infty .
$$

Relation (6) expresses weak convergence in the Skorohod space $D[0,1]$, see Billingsley [4 for basic definitions and facts for weak convergence of probability measures on metric spaces.

By a classical result of Donsker [5], if $X_{1}, X_{2}, \ldots$ are i.i.d. random variables with distribution function $F$ and $F_{N}$ denotes the empirical distribution function of the sample $\left(X_{1}, \ldots, X_{N}\right)$, then

$$
\sqrt{N}\left(F_{N}(t)-F(t)\right) \stackrel{D[0,1]}{\longrightarrow} B(F(t))
$$

where $B$ is Brownian bridge. Note the substantial difference caused by considering mod 1 sums in the present case.

If $X_{1}$ has a lattice distribution, the situation changes essentially. For example, in [1] it is shown that if $\alpha$ is irrational and $X_{1}$ takes the values $\alpha$ and $2 \alpha$ with probability $1 / 2-1 / 2$, then up to logarithmic factors, the order of magnitude of $D_{N}^{*}$ is

$$
O\left(N^{-1 / 2}\right) \text { or } O\left(N^{-1 / \gamma}\right)
$$

according as $\gamma<2$ or $\gamma>2$, where $\gamma$ is the Diophantine rank of $\alpha$, i.e., the supremum of numbers $c$ such that $|\alpha-p / q|<q^{-c-1}$ holds for infinitely many fractions $p / q$. The asymptotic distribution of $D_{N}^{*}$ in this case remains open.

\section{Proofs}

The proof of our theorems uses, similarly to that of the functional LIL in [2], a traditional blocking argument combined with a coupling lemma of Schatte, see Lemma 1 below. The substantial new difficulty is to prove the tightness of the sequence $\sqrt{N}\left(F_{N}(t)-t\right)$, since the standard maximal inequalities (e.g., Billingsley's inequalities in 4, Section 2.12) are not applicable here. We circumvent this difficulty by proving a Chernoff type exponential bound (Lemma 6) for the considered partial sums which, combined with the chaining method of Philipp [7, yields the desired fluctuation inequality (Lemma 7). 


\section{ALINA BAZAROVA — ISTVÁN BERKES — MARKO RASETA}

LEMMA 1. Let

$$
\ell \geq 1 \text { and } I_{1}, I_{2}, \ldots
$$

be disjoint blocks of integers with $\geq \ell$ integers between consecutive blocks. Then there exists a sequence $\delta_{1}, \delta_{2}, \ldots$ of random variables such that

$$
\left|\delta_{n}\right| \leq C e^{-\lambda \ell}
$$

with some positive constants $C, \lambda$ and the random vectors

$$
\left\{S_{i}, i \in I_{1}\right\}, \quad\left\{S_{i}-\delta_{1}, i \in I_{2}\right\}, \ldots,\left\{S_{i}-\delta_{n-1}, i \in I_{n}\right\}, \ldots
$$

are independent and have, except for the first one, uniformly distributed components.

Pr o of. For the proof, see 2]. The uniformity statement is implicit in the proof; see also Lemma 4.3 of [3].

In what follows, $C, \lambda, \gamma, \gamma^{\prime} \ldots$ will denote positive constants, possibly different at different places, depending (at most) on the distribution of $X_{1}$. The relation « will mean the same as the big $O$ notation, with a constant depending on the distribution of $X_{1}$.

Let $\mathcal{F}$ denote the class of functions $f$ of the form

$$
f=I_{(a, b)}-(b-a) \quad(0 \leq a<b \leq 1),
$$

extended with period 1 . For $f \in \mathcal{F}$ we put

$$
A^{(f)}:=\|f\|^{2}+2 \sum_{k=1}^{\infty} \mathbb{E} f(U) f\left(U+S_{k}\right),
$$

where $U$ is a uniform $(0,1)$ random variable, independent of $\left(X_{j}\right)_{j \geq 1}$ and $\|f\|$ denotes the $L^{2}(0,1)$ norm of $f$. Put further

$$
\widetilde{m}_{k}=\sum_{j=1}^{k}\left\lfloor j^{1 / 2}\right\rfloor, \quad \widehat{m}_{k}=\sum_{j=1}^{k}\left\lfloor j^{1 / 4}\right\rfloor
$$

and let $m_{k}=\widetilde{m}_{k}+\widehat{m}_{k}$. Using Lemma 1 we can construct sequences $\left(\Delta_{k}\right)_{k \in \mathbb{N}}$, $\left(\Pi_{k}\right)_{k \in \mathbb{N}}$ of random variables such that $\Delta_{0}=0, \Pi_{0}=0$,

$$
\left|\Delta_{k}\right| \leq C e^{-\lambda k^{1 / 4}}, \quad\left|\Pi_{k}\right| \leq C e^{-\lambda \sqrt{k}}
$$

and

$$
\begin{aligned}
T_{k}^{(f)}:=\sum_{j=m_{k-1}+1}^{m_{k-1}+\lfloor\sqrt{k}\rfloor} f\left(S_{j}-\Delta_{k-1}\right), \quad k=1,2, \ldots, \\
T_{k}^{*(f)}=\sum_{j=m_{k-1}+\lfloor\sqrt{k}\rfloor+1}^{m_{k}} f\left(S_{j}-\Pi_{k-1}\right), \quad k=1,2, \ldots
\end{aligned}
$$

are sequences of independent random variables. 
Since $\int_{0}^{1} f(x) \mathrm{d} x=0$ for $f \in \mathcal{F}$, the uniformity statement in Lemma 1 implies that

$$
\mathbb{E} T_{k}^{(f)}=\mathbb{E} T_{k}^{*(f)}=0 \quad \text { for } k \geq 2 .
$$

The following asymptotic estimates for the variances of $T_{k}^{(f)}$ and $T_{k}^{*(f)}$ are from [2].

Lemma 2. For $f \in \mathcal{F}$ we have

$$
\sum_{k=1}^{n} \operatorname{Var}\left(T_{k}^{(f)}\right) \sim A^{(f)} \widetilde{m}_{n}, \quad \sum_{k=1}^{n} \operatorname{Var}\left(T_{k}^{*(f)}\right) \sim A^{(f)} \widehat{m}_{n},
$$

where $A^{(f)}$ is defined by (17).

Since

$$
\operatorname{Cov}\left(T_{k}^{(f)}, T_{k}^{(g)}\right)=\frac{1}{4}\left(\operatorname{Var}\left(T_{k}^{(f+g)}\right)-\operatorname{Var}\left(T_{k}^{(f-g)}\right)\right),
$$

Lemma 2 implies

$$
\sum_{k=1}^{n} \operatorname{Cov}\left(T_{k}^{(f)}, T_{k}^{(g)}\right) \sim \frac{1}{4}\left(A^{(f+g)}-A^{(f-g)}\right) \widetilde{m}_{n}
$$

and

$$
\sum_{k=1}^{n} \operatorname{Cov}\left(T_{k}^{*(f)}, T_{k}^{*(g)}\right) \sim \frac{1}{4}\left(A^{(f+g)}-A^{(f-g)}\right) \widehat{m}_{n} .
$$

From (7) it follows that

$$
A^{(f+g)}-A^{(f-g)}=4\langle f, g\rangle+4 \sum_{k=1}^{\infty} \mathbb{E} f(U) g\left(U+S_{k}\right)+4 \sum_{k=1}^{\infty} \mathbb{E} g(U) f\left(U+S_{k}\right) \text {. }
$$

Lemma 3. Let $f \in \mathcal{F}, h>0$ and let $\xi$ be a random variable with $|\xi|<h$. Then for any $n \geq 1$ we have

$$
\mathbb{E}\left|f\left(S_{n}+\xi\right)-f\left(S_{n}\right)\right|^{2} \leq C h .
$$

Proof. Since $X_{1}$ is bounded with bounded density, Theorem 1 of [8] implies that the sums $S_{n}=\sum_{k=1}^{n} X_{k}(\bmod 1)$ have a uniformly bounded density and thus

$$
\mathbb{P}\left(S_{n} \in J\right) \leq C|J| \text { for any interval } J .
$$

Now if $f=I_{(a, b)}-(b-a)$, then $\left|f\left(S_{n}+\xi\right)-f\left(S_{n}\right)\right|=\left|I_{(a, b)}\left(S_{n}+\xi\right)-I_{(a, b)}\left(S_{n}\right)\right|$ is different from 0 only if one of $S_{n}+\xi$ and $S_{n}$ lies in $(a, b)$ and the other does not, which, in view of $|\xi|<h$, implies that $S_{n}$ lies closer to the boundary of $(a, b)$ than $h$, i.e., $S_{n} \in(a, a+h)$ or $S_{n} \in(b-h, b)$. Since $\left|f\left(S_{n}+\xi\right)-f\left(S_{n}\right)\right| \leq 2$, Lemma 3 follows from (12). 


\section{ALINA BAZAROVA — ISTVÁN BERKES - MARKO RASETA}

Lemma 4. For $f \in \mathcal{F}$ and any $M \geq 0, N \geq 1$ we have

$$
\mathbb{E}\left(\sum_{k=M+1}^{M+N} f\left(S_{k}\right)\right)^{2} \leq C\|f\| N
$$

Pr o of. We first show

$$
\left|\mathbb{E} f\left(S_{k}\right) f\left(S_{\ell}\right)\right| \leq C e^{-\lambda(\ell-k)}\|f\| \quad(k<\ell) .
$$

Indeed, by the proof of Lemma[1in [2], there exists a r.v. $\Delta$ with $|\Delta| \leq C e^{-\lambda(\ell-k)}$ such that $S_{\ell}-\Delta$ is a uniform r.v. independent of $S_{k}$. Hence

$$
\mathbb{E} f\left(S_{\ell}-\Delta\right)=\int_{0}^{1} f(t) \mathrm{d} t=0
$$

and thus

$$
\mathbb{E} f\left(S_{k}\right) f\left(S_{\ell}-\Delta\right)=\mathbb{E} f\left(S_{k}\right) \mathbb{E} f\left(S_{\ell}-\Delta\right)=0 .
$$

On the other hand,

$$
\begin{aligned}
& \left|\mathbb{E} f\left(S_{k}\right) f\left(S_{\ell}\right)-\mathbb{E} f\left(S_{k}\right) f\left(S_{\ell}-\Delta\right)\right| \\
& \leq \mathbb{E}\left(\left|f\left(S_{k}\right)\right|\left|f\left(S_{\ell}\right)-f\left(S_{\ell}-\Delta\right)\right|\right) \\
& \leq\left(\mathbb{E} f^{2}\left(S_{k}\right)\right)^{1 / 2}\left(\mathbb{E}\left|f\left(S_{\ell}\right)-f\left(S_{\ell}-\Delta\right)\right|^{2}\right)^{1 / 2} .
\end{aligned}
$$

Using (12) we get

$$
\mathbb{E} f^{2}\left(S_{k}\right) \leq C \int_{0}^{1} f^{2}(t) \mathrm{d} t=C\|f\|^{2} .
$$

Also, $|\Delta| \leq C e^{-\lambda(\ell-k)}$ and Lemma 3 imply

$$
\mathbb{E}\left|f\left(S_{\ell}\right)-f\left(S_{\ell}-\Delta\right)\right|^{2} \leq C e^{-\lambda(\ell-k)}
$$

which, together with (16)-(18), gives

$$
\left|\mathbb{E} f\left(S_{k}\right) f\left(S_{\ell}\right)-\mathbb{E} f\left(S_{k}\right) f\left(S_{\ell}-\Delta\right)\right| \leq C e^{-\lambda(\ell-k)} .
$$

Thus using (15) we get (14). Now by (14)

$$
\left|\sum_{M+1 \leq k<\ell \leq M+N} \mathbb{E} f\left(S_{k}\right) f\left(S_{\ell}\right)\right| \leq C N\|f\| \sum_{\ell \geq 1} e^{-\lambda \ell} \leq C N\|f\|
$$

which, together with (17), completes the proof of Lemma 4 


\section{ON THE DISCREPANCY OF RANDOM WALKS ON THE CIRCLE}

Let $0<t_{1}<\cdots<t_{r} \leq 1$ and put

where

$$
\mathbf{Y}_{k}=\left(f_{\left(0, t_{1}\right)}\left(S_{k}\right), f_{\left(0, t_{2}\right)}\left(S_{k}\right), \ldots, f_{\left(0, t_{r}\right)}\left(S_{k}\right)\right)
$$

$$
f_{(a, b)}=I_{(a, b)}-(b-a),
$$

with the indicator $I_{(a, b)}$ extended with period 1, as before.

LeMma 5. We have

where

$$
N^{-1 / 2} \sum_{k=1}^{N} \mathbf{Y}_{k} \stackrel{d}{\longrightarrow} N(\mathbf{0}, \boldsymbol{\Sigma})
$$

$$
\boldsymbol{\Sigma}=\left(\Gamma\left(t_{i}, t_{j}\right)\right)_{1 \leq i, j \leq r}
$$

Proof. Let

$$
\begin{aligned}
& \mathbf{T}_{k}=\left(T_{k}^{\left(f_{\left(0, t_{1}\right)}\right)}, \ldots, T_{k}^{\left(f_{\left(0, t_{r}\right)}\right)}\right), \\
& \mathbf{T}_{k}^{*}=\left(T_{k}^{*\left(f_{\left(0, t_{1}\right)}\right)}, \ldots, T_{k}^{*\left(f_{\left(0, t_{r}\right)}\right)}\right),
\end{aligned}
$$

and let $\boldsymbol{\Sigma}_{\mathbf{k}}$ denote the covariance matrix of the vector $\mathbf{T}_{k}$. From (9), (10) and (11) it follows that

$$
m_{n}^{-1}\left(\boldsymbol{\Sigma}_{\mathbf{1}}+\cdots+\boldsymbol{\Sigma}_{\mathbf{n}}\right) \longrightarrow \boldsymbol{\Sigma}
$$

Clearly,

$$
\left|\mathbf{T}_{k}\right| \leq C_{r} k^{1 / 2}=o\left(m_{k}^{1 / 2}\right)
$$

where $C_{r}$ is a constant depending on $r$, showing that the sequence $\left(\mathbf{T}_{k}\right)_{k \geq 1}$ of independent, mean $\mathbf{0}$ random vectors satisfies the Lindeberg condition and thus

$$
m_{n}^{-1 / 2} \sum_{k=1}^{n} \mathbf{T}_{k} \stackrel{d}{\longrightarrow} N(\mathbf{0}, \boldsymbol{\Sigma}) .
$$

A similar statement holds for the sequence $\left(\mathbf{T}_{k}^{*}\right)_{k \geq 1}$, implying that

$$
\left|\sum_{k=1}^{n} \mathbf{T}_{k}^{*}\right|=O_{P}\left(\widehat{m}_{n}\right)=o_{P}\left(m_{n}^{1 / 2}\right),
$$

and consequently,

$$
m_{n}^{-1 / 2} \sum_{k=1}^{n}\left(\mathbf{T}_{k}+\mathbf{T}_{k}^{*}\right) \stackrel{d}{\longrightarrow} N(\mathbf{0}, \boldsymbol{\Sigma}) .
$$




\section{ALINA BAZAROVA — ISTVÁN BERKES - MARKO RASETA}

Now, using (8) and Lemma 3 we get

$$
\left\|T_{k}^{(f)}-\sum_{j=m_{k-1}+1}^{m_{k-1}+\lfloor\sqrt{k}\rfloor} f\left(S_{j}\right)\right\| \ll \sqrt{k} e^{-\lambda k^{1 / 4}}
$$

and

$$
\left\|T_{k}^{*(f)}-\sum_{j=m_{k-1}+\lfloor\sqrt{k}\rfloor+1}^{m_{k}} f\left(S_{j}\right)\right\| \ll k^{1 / 4} e^{-\lambda k^{1 / 2}},
$$

and thus

$$
\left\|\sum_{k=1}^{m_{n}} \mathbf{Y}_{k}-\sum_{k=1}^{n}\left(\mathbf{T}_{k}+\mathbf{T}_{\mathbf{k}}^{*}\right)\right\|=O(1) .
$$

Together with (22) this shows that (19) holds for the indices $N=m_{n}$. To get (19) for all $N$, observe that $m_{k} \sim c k^{3 / 2}$ and thus for $m_{k} \leq N<m_{k+1}$ we have

$$
\left|\sum_{j=1}^{N} \mathbf{Y}_{j}-\sum_{j=1}^{m_{k}} \mathbf{Y}_{j}\right|=O\left(m_{k+1}-m_{k}\right)=O\left(k^{1 / 2}\right)=O\left(m_{k}^{1 / 3}\right)=O\left(N^{1 / 3}\right) .
$$

This completes the proof of Lemma 5 .

Lemma 6. For $f \in \mathcal{F}$, any $N \geq 1, t \geq 1$ and $\|f\| \geq \frac{1}{5} N^{-5 / 18}$ we have

$$
\begin{aligned}
& \mathbb{P}\left\{\left|\sum_{k=1}^{N} f\left(S_{k}\right)\right| \geq t\|f\|^{1 / 4} \sqrt{N}\right\} \ll \\
& \exp \left(-C t\|f\|^{-7 / 20}\right)+t^{-2} \exp \left(-C N^{1 / 3}\right) .
\end{aligned}
$$

REMARK. The constants $1 / 5,5 / 18,1 / 4,7 / 20,1 / 3$ in (23) are not sharp and the inequality could be easily improved. However, the present form of Lemma 6 will suffice for the chaining argument in Lemma 7.

Proof. Put

$$
\psi(n)=\sup _{0 \leq x \leq 1}\left|\mathbb{P}\left(S_{n} \leq x\right)-x\right| .
$$

By Theorem 1 of [8] we have

$$
\psi(n) \leq C e^{-\gamma n} \quad(n \geq 1)
$$

for some constant $\gamma>0$. Divide the interval $[1, N]$ into subintervals $I_{1}, \ldots, I_{L}$, with $L \sim N^{2 / 3}$, where each interval $I_{\nu}$ contains $\sim N^{1 / 3}$ terms. We set

$$
\sum_{k=1}^{N} f\left(S_{k}\right)=\eta_{1}+\cdots+\eta_{L}, \quad \text { where } \quad \eta_{\nu}=\sum_{k \in I_{\nu}} f\left(S_{k}\right)
$$




\section{ON THE DISCREPANCY OF RANDOM WALKS ON THE CIRCLE}

We deal with the sums $\sum \eta_{2 j}$ and $\sum \eta_{2 j+1}$, separately. Since there is a separation $\sim N^{1 / 3}$ between the even block sums $\eta_{2 j}$, we can apply Lemma 1 to get

where

$$
\eta_{2 j}=\eta_{2 j}^{*}+\eta_{2 j}^{* *}
$$

$$
\begin{aligned}
\eta_{2 j}^{*} & =\sum_{k \in I_{2 j}} f\left(S_{k}-\Delta_{j}\right), \\
\eta_{2 j}^{* *} & =\sum_{k \in I_{2 j}}\left(f\left(S_{k}\right)-f\left(S_{k}-\Delta_{j}\right)\right) .
\end{aligned}
$$

Here the $\Delta_{j}$ are r.v.'s with $\left|\Delta_{j}\right| \leq \psi\left(N^{1 / 3}\right) \leq C \exp \left(-\gamma N^{1 / 3}\right)$ and the r.v.'s $\eta_{2 j}^{*}$ $j=1,2, \ldots$ are independent. Conditionally on $\Delta_{j}$, the distribution of $S_{k}$ in a term of $\eta_{2 j}^{* *}$ is the same as the (unconditional) distribution of an $S_{k_{1}}+c$ with $k_{1}<k$ and a constant $c$ and thus by Lemma 3 , the $L_{2}$ norm of each summand in $\eta_{2 j}^{* *}$ is $\leq C \psi^{1 / 2}\left(N^{1 / 3}\right) \leq C \exp \left(-\gamma N^{1 / 3}\right)$ and thus for $\|f\| \geq N^{-1}$ we have

$$
\begin{aligned}
\left\|\eta_{2 j}^{* *}\right\| & \leq C N \exp \left(-\gamma N^{1 / 3}\right) \leq C\|f\| N^{2} \exp \left(-\gamma N^{1 / 3}\right) \\
& \leq C\|f\| \exp \left(-\gamma^{\prime} N^{1 / 3}\right) .
\end{aligned}
$$

Thus

$$
\left\|\sum \eta_{2 j}^{* *}\right\| \leq C\|f\| \exp \left(-\gamma^{\prime \prime} N^{1 / 3}\right)
$$

and therefore by the Markov inequality

$$
\begin{aligned}
\mathbb{P}\left(\left|\sum \eta_{2 j}^{* *}\right| \geq t\|f\|^{1 / 4} \sqrt{N}\right) & \leq C t^{-2}\|f\|^{-1 / 2} N^{-1}\|f\|^{2} \exp \left(-2 \gamma^{\prime \prime} N^{1 / 3}\right) \\
& \leq C t^{-2} \exp \left(-2 \gamma^{\prime \prime} N^{1 / 3}\right) .
\end{aligned}
$$

Let now $|\lambda| \leq d N^{-1 / 3}$ with a sufficiently small constant $d>0$. Then $\left|\lambda \eta_{2 j}^{*}\right| \leq 1 / 2$ for all $N$ and thus using $e^{x} \leq 1+x+x^{2}$ for $|x| \leq 1 / 2$ we get, using $E \eta_{2 j}^{*}=0$ for $j \geq 2$,

$$
\begin{aligned}
\mathbb{E}\left(\exp \lambda\left(\sum_{j} \eta_{2 j}^{*}\right)\right) & =\prod_{j} \mathbb{E}\left(e^{\lambda \eta_{2 j}^{*}}\right) \leq \prod_{j} \mathbb{E}\left(1+\lambda \eta_{2 j}^{*}+\lambda^{2} \eta_{2 j}^{* 2}\right) \\
& =\prod_{j}\left(1+\lambda^{2} \mathbb{E} \eta_{2 j}^{* 2}\right) \leq \exp \left(\lambda^{2} \sum_{j} \mathbb{E} \eta_{2 j}^{* 2}\right) .
\end{aligned}
$$

Here, and in the rest of the proof of the lemma, the sums and products are extended for $j \geq 2$. By Lemma 4

$$
\left\|\eta_{2 j}\right\| \leq C\|f\|^{1 / 2} N^{1 / 6}
$$

which, together with (24) and the Minkowski inequality, implies

$$
\left\|\eta_{2 j}^{*}\right\| \leq C\|f\|^{1 / 2} N^{1 / 6}
$$




\section{ALINA BAZAROVA — ISTVÁN BERKES - MARKO RASETA}

Thus the last expression in (25) cannot exceed

We choose now

$$
\exp \left(\lambda^{2} C\|f\| \sum_{j} N^{1 / 3}\right) \leq \exp \left(\lambda^{2} C\|f\| N\right) .
$$

$$
\lambda=\frac{d}{2} N^{-1 / 2}\|f\|^{-3 / 5}
$$

with the number $d$ introduced before and note that by $\|f\| \geq \frac{1}{5} N^{-5 / 18}$ we have

$$
|\lambda| \leq d N^{-1 / 3}
$$

Thus using the Markov inequality, we get

$$
\begin{aligned}
\mathbb{P}\left\{\left|\sum_{j} \eta_{2 j}^{*}\right| \geq t\|f\|^{1 / 4} \sqrt{N}\right\} & \leq 2 \exp \left\{-\lambda t\|f\|^{1 / 4} \sqrt{N}+\lambda^{2} C\|f\| N\right\} \\
& =2 \exp \left(-\|f\|^{-7 / 20} t+C\|f\|^{-1 / 5}\right) \\
& \leq 2 \exp \left(-C\|f\|^{-7 / 20} t\right) .
\end{aligned}
$$

Recall that the sum here is extended for $j \geq 2$. However, the term corresponding to $j=1$ is $O\left(N^{1 / 3}\right)$ and since $\|f\|^{1 / 4} \sqrt{N} \geq N^{0.4}$ for $N \geq N_{0}$ by the assumptions of the lemma, the last chain of estimates remains valid by including the term $j=1$ in the sum in the first probability and changing $t$ to $2 t$. A similar argument applies for the odd blocks $\eta_{2 j+1}^{*}$ (note that $\mathbb{E} \eta_{1}^{*}$ can be different from 0 , but this causes no problem), completing the proof of Lemma 6 .

Lemma 7. For any $N \geq 1,0<\delta<1$ we have

$$
\mathbb{P}\left(\sup _{0 \leq a \leq \delta}\left|\sum_{k=1}^{N}\left(I_{(0, a)}\left(S_{k}\right)-a\right)\right| \gg \delta^{1 / 8} \sqrt{N}+N^{4 / 9}\right) \ll \delta^{4}+N^{-2} .
$$

Proof. For any $h \geq 1,1 \leq j \leq 2^{h}$ let $\varphi_{h}^{(j)}$ denote the indicator function of the interval $\left[(j-1) 2^{-h}, j 2^{-h}\right)$ and put

$$
F(N, j, h)=\left|\sum_{k=1}^{N}\left(\varphi_{h}^{(j)}\left(S_{k}\right)-2^{-h}\right)\right|
$$

Clearly, $\left\|\varphi_{h}^{(j)}\right\|=2^{-h / 2}$. 


\section{ON THE DISCREPANCY OF RANDOM WALKS ON THE CIRCLE}

We observe that if $0 \leq a \leq 1$ has the dyadic expansion

$$
a=\sum_{j=1}^{\infty} \varepsilon_{j} 2^{-j}, \quad \varepsilon_{j}=0,1
$$

and $H \geq 1$ is an arbitrary integer, then $g_{a}=I_{(0, a)}$ satisfies

$$
\sum_{h=1}^{H} \varrho_{h}(x) \leq g_{a}(x) \leq \sum_{h=1}^{H} \varrho_{h}(x)+\sigma_{H}(x),
$$

where $\varrho_{h}$ is the indicator function of

and $\sigma_{H}$ is the indicator function of

$$
\left[\sum_{j=1}^{h-1} \varepsilon_{j} 2^{-j}, \sum_{j=1}^{h} \varepsilon_{j} 2^{-j}\right)
$$

$$
\left[\sum_{j=1}^{H} \varepsilon_{j} 2^{-j}, \sum_{j=1}^{H} \varepsilon_{j} 2^{-j}+2^{-H}\right) .
$$

For $\varepsilon_{h}=0$, clearly, $\varrho_{h} \equiv 0$ and thus (26) remains valid if in the sums we keep only those terms where $\varepsilon_{h}=1$. Also, for $\varepsilon_{h}=1, \varrho_{h}$ coincides with one of the $\varphi_{h}^{(j)}$ and $\sigma_{H}$ also coincides with some of the $\varphi_{H}^{(j)}$. It follows that

and

$$
\begin{aligned}
g_{a}(x)-a \leq & \sum_{1 \leq h \leq H}^{*}\left(\varrho_{h}(x)-\varepsilon_{h} 2^{-h}\right) \\
& +\left(\sigma_{H}(x)-2^{-H}\right)+2^{-H}
\end{aligned}
$$

$$
g_{a}(x)-a \geq \sum_{1 \leq h \leq H}^{*}\left(\varrho_{h}(x)-\varepsilon_{h} 2^{-h}\right)-2^{-H},
$$

where $\sum^{*}$ means that the summation is extended only for those $h$ such that $\varepsilon_{h}=1$. Setting $x=S_{k}$ and summing for $1 \leq k \leq N$ we get

$$
\begin{aligned}
\sum_{k \leq N}\left(g_{a}\left(S_{k}\right)-a\right) \leq \sum_{k \leq N} & \sum_{1 \leq h \leq H}^{*}\left(\varrho_{h}\left(S_{k}\right)-\varepsilon_{h} 2^{-h}\right) \\
& \quad+\sum_{k \leq N}\left(\sigma_{H}\left(S_{k}\right)-2^{-H}\right)+N 2^{-H}
\end{aligned}
$$

and

$$
\sum_{k \leq N}\left(g_{a}\left(S_{k}\right)-a\right) \geq \sum_{k \leq N} \sum_{1 \leq h \leq H}^{*}\left(\varrho_{h}\left(S_{k}\right)-\varepsilon_{h} 2^{-h}\right)-N 2^{-H} .
$$




\section{ALINA BAZAROVA — ISTVÁN BERKES - MARKO RASETA}

Hence it follows that for any $N \geq 1, H \geq 1$ there exist suitable integers $1 \leq j_{h} \leq 2^{h}$, $1 \leq h \leq H$ such that

Introduce the events

$$
\begin{aligned}
\left|\sum_{k \leq N}\left(g_{a}\left(S_{k}\right)-a\right)\right| & \leq 2 \sum_{h \leq H}\left|\sum_{k \leq N} \varphi_{h}^{\left(j_{h}\right)}\left(S_{k}\right)-2^{-h}\right|+N 2^{-H} \\
& =2 \sum_{h \leq H} F\left(N, j_{h}, h\right)+N 2^{-H} .
\end{aligned}
$$

$$
\begin{aligned}
G(N, j, h) & =\left\{F(N, j, h) \geq 2^{-h / 8} \sqrt{N}\right\}, \\
G_{N} & =\bigcup_{A \leq h \leq B \log _{2} N} \bigcup_{j \leq 2^{h}} G(N, j, h) \text { with } A=\log _{2} \frac{1}{a}, \quad B=\frac{5}{9} .
\end{aligned}
$$

For $h \leq B \log _{2} N$ we have

$$
\left\|\varphi_{j}^{(h)}-2^{-h}\right\| \geq 2^{-h / 2}-2^{-h} \geq 2^{-h / 2}(1-1 / \sqrt{2}) \geq \frac{1}{5} N^{-5 / 18}
$$

and thus applying (23) with $t=1$, we get

$$
\mathbb{P}(G(N, h, j)) \ll \exp \left(-C 2^{7 h / 40}\right)+N^{-3},
$$

and consequently,

$$
\mathbb{P}\left(G_{N}\right) \ll \sum_{h=A}^{\infty} 2^{h} \exp \left(-C 2^{7 h / 40}\right)+N^{-3} \sum_{h \leq B \log _{2} N} 2^{h} .
$$

Clearly, the second term on the right-hand side of (28) is $\ll N^{-2}$. On the other hand, the terms of the first sum in (28) decrease superexponentially and thus the sum can be bounded by a constant times its first term, i.e., the sum is

Hence

$$
\ll 2^{A} \exp \left(-C 2^{7 A / 40}\right) \ll 2^{-4 A} \ll a^{4} .
$$

$$
\mathbb{P}\left(G_{N}\right) \ll a^{4}+N^{-2} .
$$

Note that when breaking the interval $(0, a)$ into dyadic intervals of length $2^{-h}$ we automatically have $h \geq \log \frac{1}{a}=A$ and thus choosing

$$
H=\left[B \log _{2} N\right]
$$

it follows that with the exception of a set with probability $\ll a^{4}+N^{-2}$, for any $0<a \leq \delta$ the expression in the second line of (27) is

$$
\begin{aligned}
\ll \sum_{A \leq h \leq H} 2^{-h / 8} \sqrt{N}+N 2^{-H} & \ll 2^{-A / 8} \sqrt{N}+N^{4 / 9} \ll \\
a^{1 / 8} \sqrt{N}+N^{4 / 9} & \ll \delta^{1 / 8} \sqrt{N}+N^{4 / 9} .
\end{aligned}
$$

This proves Lemma 7 . 


\section{ON THE DISCREPANCY OF RANDOM WALKS ON THE CIRCLE}

Lemma 5 implies the convergence of the finite dimensional distributions of the sequence $\sqrt{N}\left(F_{N}(t)-t\right)$ in (5) to those of $K$ and to prove Theorem 2 it remains to prove the tightness of the sequence in $D[0,1]$. To this end, fix $\varepsilon>0$ and choose $h$ so that $2^{-h} \leq \varepsilon<2^{-(h-1)}$. Note that for $j=0,1, \ldots, 2^{h}-1$ we have

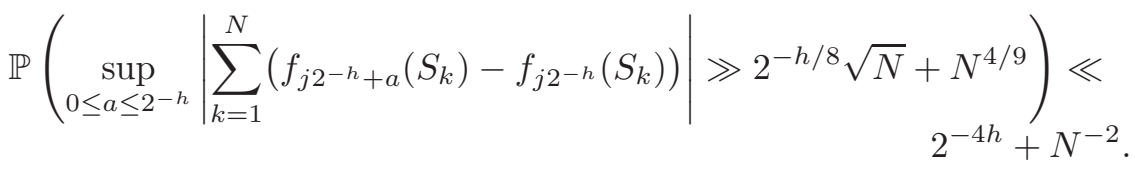

For $j=0$ relation (29) is identical with Lemma 7 and for $j=1,2, \ldots$ the proof is the same. It follows that

$$
\mathbb{P}\left(\max _{0 \leq j \leq 2^{h}-1} \sup _{0 \leq a \leq 2^{-h}}\left|\sum_{k=1}^{N}\left(f_{j 2^{-h}+a}\left(S_{k}\right)-f_{j 2^{-h}}\left(S_{k}\right)\right)\right| \gg 2^{-h / 8} \sqrt{N}+N^{4 / 9}\right) \ll
$$

Then (30) implies that with the exception of a set with probability

$$
\ll 2^{-3 h}+2^{h} N^{-2} \ll \varepsilon^{3}+N^{-2} \varepsilon^{-1}
$$

the fluctuation of the process $\sqrt{N}\left(F_{N}(t)-t\right)$ over any subinterval of $(0,1)$ with length $\leq \varepsilon$ is

$$
\ll \varepsilon^{1 / 8}+N^{-1 / 18} \text {. }
$$

By Theorem 15.5 of Billingsley [4, p. 127], the sequence $\sqrt{N}\left(F_{N}(t)-t\right)$ is tight in $D[0,1]$. This completes the proof of Theorem [2, Theorem 1 follows immediately from Theorem 2 .

ACKNOWLEDGEMENTS. The authors are grateful to the referee for several useful comments leading to a substantial improvement of the paper.

\section{REFERENCES}

[1] BERKES, I.-BORDA, B.: On the discrepancy of random subsequences of $\{n \alpha\}$, Acta Arithmetica (to appear).

[2] BERKES, I.-RASETA, M.: On the discrepancy and empirical distribution function of $\left\{n_{k} \alpha\right\}$, Unif. Distr. Theory 10 (2015), no. 1, 1-17.

[3] BERKES, I.-WEBER, M.: On the convergence of $\sum c_{k} f\left(n_{k} x\right)$, Mem. Amer. Math. Soc. 201 (2009), no. 943, viii+72 pp.

[4] BILlingSLEY, P.: Convergence of probability measures. Wiley, 1968.

[5] DONSKER, M.: Justification and extension of Doob's heuristic approach to the Kolmogorov-Smirnov theorems, Ann. Math. Statist. 23 (1952), 277-281. 


\section{ALINA BAZAROVA — ISTVÁN BERKES - MARKO RASETA}

[6] LÉVY, P.: L'addition des variables aléatoires définies sur une circonference, Bull. Soc. Math. France 67 (1939), 1-40.

[7] PHILIPP, W.: Limit theorems for lacunary series and uniform distribution mod 1, Acta Arith. 26 (1975), 241-251.

[8] SCHATTE, P.: On the asymptotic uniform distribution of sums reduced mod 1, Math. Nachr. 115 (1984), 275-281.

[9] SCHATTE, P.: On a uniform law of the iterated logarithm for sums mod 1 and Benford's law, Lithuanian Math. J. 31 (1991), 133-142.

Received August 24, 2018

Accepted March 12, 2019

\author{
Alina Bazarova \\ Institute for Biological Physics \\ University of Cologne \\ Zülpicher Straße 77 \\ D-50937 Köln \\ GERMANY \\ E-mail: a.bazarova@uni-koeln.de
}

\section{István Berkes}

Rényi Institute of Mathematics

Reáltanoda u. 13-15

H-1053 Budapest

HUNGARY

E-mail: berkes.istvan@renyi.mta.hu

\section{Marko Raseta}

Research Institute for Primary Care and Health Sciences and Research Institute for Applied Clinical Sciences

University of Keele

Keele ST5 5BG, Staffordshire

UNITED KINGDOM

E-mail:m.raseta@keele.ac.uk 